\title{
AVALIAÇÃO NUTRICIONAL SUBJETIVA. Parte 1 - Revisão de sua validade após duas décadas de uso
}

\author{
Maria Cristina Gonzalez BARBOSA-SILVA e Aluísio Jardim Dornellas de BARROS
}

RESUMO - Racional - A avaliação nutricional subjetiva é método clínico de avaliação do estado nutricional, que considera não apenas alterações da composição corporal, mas também alterações funcionais do paciente. É método simples, de baixo custo e não-invasivo, podendo ser realizado à beira do leito. Seu uso foi descrito pela primeira vez há quase duas décadas. Objetivos Revisar os estudos de validação da avaliação nutricional subjetiva descritos na literatura nas últimas duas décadas de seu uso. Métodos - Foi realizada uma revisão sistemática no MEDLINE utilizando o descritor "subjective global assessment" e selecionados os trabalhos mais relevantes. Resultados - Por se tratar de método subjetivo, sua precisão depende da experiência do observador. Porém, quando usado por observadores experientes, apresenta boa precisão diagnóstica. A avaliação nutricional subjetiva foi legitimada através da validação convergente, onde o método foi comparado com outros métodos objetivos de avaliação nutricional, e da validação preditiva, mostrando que a técnica identificou pacientes de alto risco para desenvolver complicações pós-operatórias. Conclusão - A avaliação nutricional subjetiva tem se mostrado boa opção na avaliação nutricional de pacientes cirúrgicos e algumas modificações têm sido sugeridas para o uso do método também em outras especialidades. A experiência do observador é de extrema importância, uma vez que dela depende a precisão do método.

DESCRITORES - Avaliação nutricional. Transtornos nutricionais.

\section{INTRODUÇÃO}

O estudo da prevalência de desnutrição no ambiente hospitalar tem tido destaque nos últimos 20 anos, e trabalhos em todo o mundo têm mostrado prevalências que variam de $30 \%$ a $50 \%$ em pacientes clínicos e cirúrgicos. Entretanto, diferentes critérios e diferentes métodos foram utilizados nestes estudos, devido à falta de um conceito universal para definir desnutrição ${ }^{(11)}$. O termo "desnutrição protéico-calórica" referese a um antigo conceito baseado principalmente em medidas objetivas do estado nutricional, tais como avaliação da ingestão oral, peso corporal e perda de peso, antropometria, dosagem de proteínas hepáticas, avaliação da imunidade celular e avaliação da composição corporal. Embora estes indicadores sejam úteis em estudos epidemiológicos de desnutrição, nenhuma medida isoladamente pode ser considerada válida para avaliação individual do paciente hospitalizado ${ }^{(7,37,42)}$. Todos apresentam limitações, sendo a mais importante o fato de serem influenciados por fatores independentes do estado nutricional ${ }^{36}$
37). Testes precisos, considerados como padrão ouro para avaliação da composição corporal, como medida de potássio corporal total $\left({ }^{40} \mathrm{~K}\right)$ para quantificar a massa celular corporal ou nitrogênio corporal total para avaliação do conteúdo protéico corporal, têm seu uso limitado na prática clínica devido ao custo e à pouca praticidade dos métodos, ficando restritos a ambientes de pesquisa ${ }^{(13,42)}$.

No ambiente hospitalar, desnutrição seria mais bem descrita como processo contínuo que se desencadeia com a ingestão inadequada de nutrientes em relação as suas necessidades e progride através de uma seqüência de alterações funcionais que precedem as alterações na composição corporal $^{(32)}$. ALLISON ${ }^{(2)}$ sugere uma definição baseada na repercussão clínica, onde desnutrição seria um estado de deficiência energética, protéica ou de qualquer outro nutriente específico, que implicasse em alteração mensurável de função corporal, associada a uma pior evolução da doença e que fosse especificamente reversível pela terapia nutricional. A presença de alterações funcionais parece ser um fator mais importante

Departamento de Medicina Social, Faculdade de Medicina, Universidade Federal de Pelotas e Santa Casa de Misericórdia de Pelotas, RS, Brasil.

Trabalho realizado sob patrocínio da Fundação Coordenação de Aperfeiçoamento de Pessoal de Nível Superior - CAPES

Endereço para correspondência: Dra. Maria Cristina Gonzalez Barbosa e Silva - Rua Ariano de Carvalho, 304 - $96055-800$ - Pelotas, RS, Brasil. e-mail: mcsilva@epidemioufpel.org.br. 
na ocorrência de complicações associadas à desnutrição do que a presença de alterações da composição corporal isoladamente ${ }^{(28)}$. Estas alterações se manifestam principalmente em distúrbios funcionais no trato digestivo, sistema imune e função muscular, que normalmente são a causa de morbimortalidade destes pacientes ${ }^{(26)}$. Sendo assim, a avaliação nutricional ideal no paciente hospitalizado deveria ter a sensibilidade de detectar alterações funcionais orgânicas que ocorrem precocemente no processo de desnutrição. Sob este enfoque, a desnutrição seria identificada previamente ao aparecimento de alterações na composição corporal associada à carência de nutrientes.

A partir dessa associação surgiu o conceito de "complicações associadas ao estado nutricional"(20), ou seja, risco aumentado de morbimortalidade em decorrência do seu estado nutricional (freqüentemente denominado "risco nutricional"). Desta forma, mais importante do que o diagnóstico de desnutrição seria a avaliação do risco de deterioração nutricional naqueles pacientes em situações que podem estar associadas a problemas nutricionais ${ }^{(37)}$. JEEJEEBHOY ${ }^{(32)}$ sugere que o método ideal de avaliação do estado nutricional, para que tenha importância clínica, deve ser capaz de predizer a ocorrência de complicações associadas ao estado nutricional. Alguns autores diferenciam este processo de identificação de risco nutricional, denominando-o de método de rastreamento de risco nutricional ${ }^{(10)}$

BAKER et al. ${ }^{(3)}$ validaram o uso da avaliação clínica como método capaz de identificar pacientes cirúrgicos de risco nutricional. Este método clínico obteve boa correlação com a morbidade pós-operatória, assim como com os dados antropométricos e laboratoriais comumente utilizados para a avaliação nutricional. DETSKY et al. ${ }^{(18)}$ padronizaram este método essencialmente clínico, criando uma versão em forma de questionário, denominado avaliação subjetiva global ou avaliação nutricional subjetiva (ANS) do estado nutricional (Figura 1).

\section{Avaliação subjetiva global do estado nutricional}

(Selecione a categoria apropriada com um X ou entre com valor numérico onde indicado por '“\#')

\section{A. História}

1. Alteração no peso

Perda total nos últimos 6 meses: total = \# kg; $\%$ perda $=\#$

Alteração nas últimas duas semanas: aumento sem alteração diminuição.

2. Alteração na ingestão alimentar sem alteração alterada duração $=$ \# semanas tipo:____ dieta sólida sub-ótima ___ dieta líquida completa líquidos hipocalóricos inanição.

3. Sintomas gastrintestinais (que persistam por $>2$ semanas)

_ nenhum ___ náusea __ vômitos___ diarréia ___ anorexia.

4. Capacidade funcional sem disfunção (capacidade completa) disfunção ___ duração = \# semanas. tipo: trabalho sub-ótimo___ambulatório acamado.

5. Doença e sua relação com necessidades nutricionais
Diagnóstico
primário

(especificar)

Demanda metabólica (stress): sem stress baixo stress stress moderado stress elevado.

B. Exame Físico (para cada categoria, especificar: $0=$ normal, $1+=$ leve, $2+=$ moderada, $3+=$ grave)

\# ___ perda de gordura subcutânea (tríceps, tórax)

\# ___ perda muscular (quadríceps, deltóide)

\#___edema tornozelo

\# ___ edema sacral

\# ascite

C. Avaliação subjetiva global (selecione uma) $\mathrm{A}=$ bem nutrido

$\mathrm{B}=$ moderadamente (ou suspeita de ser) desnutrido

$\mathrm{C}=$ gravemente desnutrido

FIGURA 1 - Avaliação subjetiva global segundo DETSKY et al. ${ }^{(18)}$ 
Este método simples, de baixo custo, que pode ser realizado em poucos minutos à beira do leito, foi inicialmente motivo de controvérsia, uma vez que sugeria que o julgamento clínico fosse superior a medidas objetivas comumente utilizadas ${ }^{(25)}$. Após sua comparação com métodos considerados padrões ouro, a ANS obteve grande aceitação na prática clínica, sendo atualmente utilizado não apenas em pacientes cirúrgicos, mas também adaptado para várias outras situações clínicas. Desde sua publicação inicial em 1987, vários autores têm referido a utilização deste método, sendo avaliada sua repetibilidade, validades convergente e preditiva e seu uso em estudos de intervenção. Os principais resultados destes trabalhos foram analisados para a elaboração desta revisão.

\section{Vantagens e desvantagens do método}

A ANS tem sido amplamente utilizada, por se tratar de método de fácil execução, dispensando recursos dispendiosos e podendo ser realizado por profissionais não-médicos de equipe multidisciplinar de terapia nutricional. Estudos comparativos mostraram existir associação significativa da ANS com os métodos objetivos usados na avaliação nutricional ${ }^{(3,9,17,18,21,29,40)}$. A ANS torna-se útil não só para uso hospitalar, mas também para monitorar pacientes domiciliares $^{(21,30)}$, ou estudos multicêntricos, tornando possível a padronização da avaliação nutricional em locais de recursos técnicos diferentes ${ }^{(8,12)}$.

Por combinar informações sobre alterações na ingestão de nutrientes, digestão e absorção, mas também seus efeitos na função e na composição corporal, os resultados encontrados pela ANS podem diferir daqueles encontrados por outros métodos objetivos, como índices nutricionais ou perda de peso isoladamente ${ }^{(12,38)}$. Segundo DETSKY et al. ${ }^{(15)}$ e DETSKY ${ }^{(19)}$, o propósito da realização da avaliação nutricional não seria apenas o diagnóstico, mas sim a maneira de identificar aqueles pacientes com maior risco de complicações associadas ao estado nutricional durante sua internação (avaliação de risco nutricional). Desta forma, a avaliação nutricional seria instrumento tanto prognóstico, como diagnóstico. A capacidade prognóstica do teste foi demonstrada em vários estudos, nos quais os pacientes identificados como desnutridos graves pela ANS tiveram mais complicações infecciosas ${ }^{(3,15,19)}$, maior mortalidade ${ }^{(14,24)}$ e maiores custos hospitalares $^{(5)}$. Por outro lado, apenas os pacientes considerados desnutridos graves pela ANS se beneficiaram da terapia nutricional pré-operatória, em estudo multicêntrico realizado em pacientes cirúrgicos $^{(43)}$. Evidenciou-se também que a habilidade da ANS em prever complicações depende da população estudada ${ }^{(31)}$. Em pacientes clínicos, NABER et al. ${ }^{(38)}$ demonstraram que aqueles desnutridos graves tiveram maior risco de complicações, porém fatores como idade, tipo e gravidade de doença podem ter confundido o efeito da desnutrição.
No ambiente hospitalar, o que se denomina "desnutrição" talvez seja determinado por diversos fatores e não simplesmente a falta de ingestão adequada de calorias, proteínas e micronutrientes. Assim, o diagnóstico de "desnutrição" através da ANS seria, na verdade, um marcador do "estado de saúde", sendo a desnutrição grave indicador da gravidade da doença, e não apenas indicador da magnitude do déficit de nutrientes ${ }^{(14,19,20,31)}$. Isto explicaria a capacidade preditiva do método e também a incapacidade de se demonstrar claramente a diminuição de risco nos pacientes desnutridos graves apenas com o uso da terapia nutricional ${ }^{(19)}$

Outra limitação do método é sua utilização para monitorar a evolução dos pacientes. Como a ANS é baseada exclusivamente em critérios qualitativos, pequenas alterações do estado nutricional não seriam detectadas, embora os demais métodos objetivos utilizados na prática clínica para avaliação do estado nutricional também não possuam sensibilidade ou precisão suficientes para detectar pequenas variações no estado nutricional, ocorridas em curto prazo ${ }^{(29)}$. No entanto, BRAUNSCHWEIG(5) e BRAUNSCHWEIG et al. ${ }^{(6)}$ podem demonstrar que os pacientes que apresentaram piora do seu estado nutricional, segundo a ANS de internação e de alta, tiveram os custos hospitalares significativamente mais altos e maior incidência de complicações que aqueles que mantiveram seu estado nutricional. Nestes estudos, porém, foram incluídos apenas pacientes com internação superior a 7 dias, sendo este o intervalo mínimo para que a avaliação fosse repetida.

\section{Repetibilidade}

Por se tratar de método subjetivo, a ANS tem sua precisão diagnóstica dependente da experiência do observador, sendo esta sua principal desvantagem. Em trabalho inicial, BAKER et al. ${ }^{(3)}$ obtiveram boa concordância entre seus observadores (kappa $=0,72$ ). Posteriormente, DETSKY et al. ${ }^{(17,18)}$ encontraram boa concordância quando a avaliação foi realizada por dois médicos $(k a p p a=0,78)$ e concordâncias variáveis quando foi realizada por residentes e enfermeiras treinadas (kappa de 0,6 a 1,0). Em estudo de validação, posteriormente realizado por HIRSCH et al. ${ }^{(29)}$, a concordância obtida por um médico residente e um especialista foi considerada boa (kappa $=0,66$ ), sendo que o primeiro subestimou o diagnóstico de desnutrição, quando comparado com o segundo.

Outros autores ${ }^{(41)}$, utilizando o método em outras populações de pacientes, também obtiveram bons resultados de precisão deste. A avaliação pré-operatória através da ANS de pacientes pré-transplante hepático, mostrou que houve discordância no diagnóstico em apenas um paciente, sendo os dois observadores experientes com o método.

$\mathrm{Na}$ avaliação de pacientes geriátricos, os resultados têm se mostrado controversos. COVINSKY et al. ${ }^{(14)}$ obtiveram boa concordância $(k a p p a=0,71)$ quando a avaliação foi realizada 
concomitantemente por um médico internista e por uma enfermeira, ambos com treinamento na prática de avaliação nutricional. No entanto, em estudo realizado em pacientes idosos, EK et al. ${ }^{(22)}$ não conseguiram obter valores de concordância satisfatórios entre seus observadores, quando apenas um deles tinha experiência com avaliação nutricional com métodos objetivos e subjetivos. Neste estudo obteve kappa $=$ 0,56 , sendo este valor sugestivo de precisão inadequada para utilização do método em pesquisa. Uma explicação para este resultado pode ser o diferente grau de experiência dos dois observadores, sendo que apenas o observador mais experiente obteve boa concordância com os métodos objetivos e ANS. Mais uma vez se reforça a idéia de que a precisão do método depende mais da experiência e treinamento dos observadores na identificação dos aspectos significativos da avaliação nutricional, do que de características específicas das populações em estudo. CORREIA ${ }^{(12)}$, em seu estudo multicêntrico IBRANUTRI, demonstrou que com treinamento adequado pode-se conseguir boa concordância entre diferentes avaliadores. Obteve kappas que variaram de 0,78 a 0,83 entre três observadores avaliados.

Outros autores, na tentativa de tornar o método mais preciso, modificaram a ANS através de índices numéricos fornecidos a cada item, na tentativa de transformá-la num método quantitativo. O método subjetivo original foi modificado tanto para uso geral, na população hospitalar $^{(9)}$, como para uso em pacientes nefropatas ${ }^{(35)}$ ou hepatopatas $^{(27)}$. Após a utilização da ANS modificada, KALANTAR-ZADEH et al. ${ }^{(34)}$ encontraram concordância muito boa entre seus avaliadores (kappa = 0,83 ), sendo a avaliação realizada por um médico e uma nutricionista.

Em relação a repetibilidade, pode-se concluir que sendo realizada por avaliadores bem treinados, pode-se obter bons resultados, comparáveis aos métodos objetivos comumente utilizados.

\section{Validade Convergente}

$\mathrm{Na}$ falta de um teste de referência para diagnóstico de desnutrição, diferentes estratégias são utilizadas para validar novas técnicas de avaliação nutricional. Uma delas é a chamada validação convergente, em que o resultado da nova técnica é comparado com outras já comprovadas e os resultados devem convergir para a mesma resposta. Em seu estudo inicial, BAKER et al. ${ }^{(3)}$ compararam os resultados da ANS com as técnicas de avaliação nutricional objetivas, como dosagem de albumina sérica, transferrina, linfócitos totais, percentual de massa magra atual/massa magra ideal, percentual de peso atual/peso ideal, índice creatinina-altura, percentual de gordura corporal, nitrogênio e potássio corporal total e provas de sensibilidade cutânea. A análise de variância foi significativa para as médias entre as categorias A, B e C da maioria dos parâmetros objetivos estudados, exceto transferrina, linfócitos totais e nitrogênio corporal total. Através da análise de Newman-Keuls, foram identificadas diferenças entre os valores médios das categorias A e C ou B e C para a maioria dos parâmetros analisados, mas nenhum parâmetro mostrou diferença entre as médias das categorias A e B. Estes resultados foram confirmados em estudo posterior do mesmo grupo ${ }^{(17)}$

Após estes estudos iniciais, vários autores ${ }^{(9,21,29)}$ comprovaram a existência de valores médios de testes bioquímicos e antropométricos significativamente diferentes entre as três categorias da ANS. No Inquérito Brasileiro de Desnutrição Hospitalar (IBRANUTRI), estudo multicêntrico realizado em nosso meio, também foi encontrada associação entre valores baixos de índice de massa corporal e albumina sérica e desnutrição, de acordo com a $\mathrm{ANS}^{(44)}$.

Em estudo comparativo entre ANS e outros índices nutricionais, como o índice de risco nutricional (IRN) e índice de Maastricht (IM), ambos obtidos a partir de parâmetros objetivos como albumina sérica, percentual peso atual/peso usual, transtirretina e linfócitos, foram encontrados resultados diferentes entre os três métodos. Menor prevalência de desnutrição foi encontrada pela ANS, quando comparada com o IRN e IM. No entanto, além de utilizar população clínica, os autores utilizaram uma classificação diferente da proposta por BAKER et al. ${ }^{(3)}$, visto que foi criada a categoria de "desnutrição leve", inexistente na ANS original ${ }^{(38)}$.

Em pacientes nefropatas, apesar das limitações do uso dos parâmetros laboratoriais e antropométricos presentes, também foi encontrada convergência de resultados em alguns estudos realizados. Em estudo com pacientes em esquema de hemodiálise ou diálise peritonial ambulatorial crônica por pelo menos 4 meses, foi demonstrado que a albumina sérica e o ângulo de fase obtido pela bioimpedância elétrica foram significativamente diferentes entre os pacientes considerados nutridos ou desnutridos pela ANS. A análise univariada mostrou correlação negativa entre estes parâmetros e as categorias da ANS, sendo a correlação de $r=-0,58$ para o ângulo de fase e $r=-0,51$ para a albumina. A correlação obtida entre os diversos parâmetros objetivos utilizados e a ANS mostrou ser, no mínimo, comparável àquela obtida entre os parâmetros objetivos ${ }^{(23)}$. Em estudo posterior, JONES et al. ${ }^{(33)}$ também encontrou valores médios menores de peso atual, índice de massa corporal, massa corporal magra, circunferência muscular do braço e força muscular em pacientes considerados desnutridos pela ANS, numa população de pacientes renais em CAPD.

KALANTAR-ZADEH et al. ${ }^{(34)}$ estudaram a capacidade total de ligação ao ferro (CTLF) como um dos parâmetros laboratoriais utilizados para se estimar a transferrina e sua associação com a ANS. Seus resultados mostraram que a CTLF obtida era significativamente diferente entre as três categorias de estado nutricional diagnosticado pela ANS. Esta relação entre os valores de CTLF e estado nutricional, ou seja, menores valores em pacientes com pior estado nutricional, mostrou-se independente da idade, tempo de tratamento e dose de eritropoetina. Neste mesmo estudo, parâmetros como a albumina e ferritina apenas se mostraram significativamente diferentes naqueles pacientes classificados como desnutridos graves pela ANS. 
Além de apresentar validação convergente com parâmetros objetivos utilizados na avaliação nutricional, a ANS também se mostrou associada com citocinas catabólicas e fatores de crescimento anabólicos $^{(1)}$. Em estudo com 20 pacientes renais crônicos, foram encontrados valores significativamente menores de IGF-1 (fator de crescimento semelhante à insulina com propriedades anabólicas) e valores significativamente maiores de TNF- $\alpha$ (fator de necrose tumoral $\alpha$, citocina pró-inflamatória catabólica) entre aqueles pacientes considerados desnutridos pela ANS.

Também em pacientes com insuficiência renal aguda, FIACCADORI et al. ${ }^{(24)}$ encontraram diferenças significativas entre os valores médios da prega cutânea do tríceps, área muscular do braço, albumina, transferrina, pré-albumina e linfócitos totais entre as três categorias da ANS.

Em pacientes com infecção por HIV, BOWERS e DOLS ${ }^{(4)}$ demonstraram existir associação significativa com tendência linear entre as categorias de ANS e albumina sérica $<3,5 \mathrm{~g} / \mathrm{dL}$ e linfócitos CD4 $\leq 100 \mu \mathrm{L}$ : enquanto nenhum paciente considerado bem nutrido pela ANS possuía albumina ou linfócitos CD4 alterados, nos pacientes desnutridos graves $63,6 \%$ possuíam albumina alterada e $90,9 \%$, linfócitos CD4 diminuídos. Este trabalho sugere com estes resultados, que a albumina sérica não é parâmetro sensível para detectar desnutrição no paciente HIV positivo, vindo a se alterar tardiamente na evolução da doença. Em estudo comparativo da ANS com a classificação do sistema CDC (Centers for Disease Control and Prevention) para pacientes infectados por HIV, foi encontrada associação significativa entre as suas categorias ${ }^{(39)}$. Nenhum paciente classificado como A (paciente assintomático) ou B (pouco sintomático) pelo CDC foi classificado como desnutrido grave, demonstrando boa especificidade do método. Todos os pacientes classificados como desnutridos graves estavam na categoria $\mathrm{C}$ pelo CDC (com AIDS), porém isto representava apenas $14 \%$ dos pacientes com AIDS (pouca sensibilidade). Em relação às variáveis antropométricas e da bioimpedância elétrica, também foram encontradas diferenças significativas nos valores médios entre as categorias da ANS, comprovando a validade convergente do método nesta situação clínica.

Em pacientes geriátricos, EK et al. ${ }^{(22)}$ demonstraram validação convergente entre as variáveis antropométricas e a ANS, porém os valores de albumina sérica e transtirretina não mostraram diferença significativa entre os pacientes considerados desnutridos ou não pela ANS.

A maioria dos trabalhos mostrou que existe concordância entre o diagnóstico de desnutrição realizado pela ANS e através de parâmetros objetivos, como variáveis antropométricas e testes laboratoriais, confirmando sua validação convergente. Entretanto, alguns estudos utilizaram técnicas que não seriam as mais adequadas para avaliar esta concordância. Uma vez que os parâmetros objetivos foram utilizados como variáveis contínuas, na maioria das vezes, o ideal teria sido a realização de análise de variância destes parâmetros entre as três categorias da ANS, e não correlação, considerando a ANS uma variável contínua.

\section{Validade Preditiva}

Os métodos de avaliação nutricional em pacientes hospitalizados têm sido utilizados não apenas com o objetivo de diagnosticar o estado nutricional per se, mas também para identificar aqueles pacientes que se encontram sob maior risco de desenvolverem complicações durante sua internação. Esta associação entre estado nutricional e complicações é denominada "risco nutricional", sendo que em pacientes cirúrgicos pode incluir deiscência de suturas e infecções ${ }^{(19)}$. Para ser usada com este objetivo, no qual a ANS deixa de ser método diagnóstico e passa a ser considerado método de prognóstico clínico, a ANS deveria ser submetida a testes de validação preditiva, ou seja, estudos que demonstrem que o método identifica adequadamente os pacientes que possuem maior risco de apresentarem complicações.

O primeiro estudo que demonstrou a validade preditiva da ANS foi o de BAKER et al. ${ }^{(3)}$, onde se mostrou que os pacientes considerados desnutridos graves pela ANS tiveram maior incidência de infecção, maior uso de antibióticos e maior internação hospitalar. Numa população maior, de 202 pacientes cirúrgicos, DETSKY et al. ${ }^{(17)}$ demonstraram que a ANS teria sua capacidade preditiva aumentada quando associada à dosagem sérica pré-operatória da albumina.

Apesar das vantagens associadas ao uso da ANS como método preditivo, autores chamam a atenção de que a habilidade do método depende também da população estudada ${ }^{(31)}$. Os melhores resultados preditivos (probabilidade pós-teste) são obtidos com a combinação do resultado da ANS com a probabilidade pré-teste, que depende das circunstâncias clínicas de cada doente ${ }^{(16)}$.

NABER et al. ${ }^{(38)}$ realizaram estudo com pacientes clínicos, comparando a validade preditiva da ANS e outros índices nutricionais. Seus achados sugerem que, após correção para fatores de confusão, tais como capacidade funcional, número de drogas usadas, duração da internação, categoria da doença e cirurgia, a razão de odds (RO) da ANS para complicações torna-se muito pequena $(\mathrm{RO}=1,2$ para complicações severas a $\mathrm{RO}=1,9$ para complicações não severas) e deixa de ser significativa. No entanto, existe a possibilidade de erro de classificação neste estudo, uma vez que foi utilizada categoria denominada "desnutrição leve", inexistente na ANS original, que corresponde a quase metade dos pacientes considerados com algum grau de desnutrição. Desta maneira, a ANS pode ter tido sua capacidade preditiva subestimada. PIKUL et al. ${ }^{(41)}$ também utilizaram a ANS com quatro categorias, incluindo desnutrição leve, numa coorte retrospectiva de pacientes submetidos a transplantes hepáticos. Nesse estudo, apenas foram capazes de encontrar diferenças significativas entre as médias de dias de internação em UTI, dias de ventilação e dias de hospitalização entre os pacientes considerados desnutridos moderados e graves, quando comparados aos desnutridos leves e normais. Estes estudos reforçam a necessidade de utilizar a classificação original da ANS, para que não ocorra erro de classificação do estado nutricional, diminuindo, assim, as associações que poderiam ser encontradas. 
Em pacientes nefropatas, mostrou-se que além de validade convergente a ANS também apresentou validade preditiva ${ }^{(24)}$. Houve aumento progressivo da mortalidade e morbidade entre as categorias A, B e C da ANS (com tendência linear significativa), tanto para as complicações infecciosas, como para as não-infecciosas. A desnutrição grave pré-existente foi considerada variável preditora independente para mortalidade hospitalar na regressão logística múltipla, mesmo quando ajustado para outros variáveis de comorbidade $(\mathrm{RO}=2,02$; IC: 1,50 a 2,71) e complicações ( $\mathrm{RO}=2,12$; IC: 1,61 a 2,89).

Em estudo longitudinal com pacientes idosos internados, sendo avaliados durante a internação, 3 e 12 meses após a alta hospitalar, a ANS mostrou ter validade preditiva não apenas para a mortalidade após alta hospitalar, mas também para desfechos representativos da recuperação funcional destes pacientes, como dependência para atividades cotidianas diárias e internação em clínicas de repouso ${ }^{(14)}$. Foi encontrado aumento progressivo da mortalidade em 3 e 12 meses de acordo com o estado nutricional definido pela ANS. Em pacientes considerados desnutridos graves, mesmo após ajuste para variáveis como características demográficas, gravidade da doença, condições de co-morbidade, e número de atividades diárias dependentes, foram encontrados valores de $\mathrm{RO}=3,26$ (IC: 1,52 a 6,96) para mortalidade em 3 meses pós-alta hospitalar (tendência linear com $P=0,003$ ), e $\mathrm{RO}=2,83$ (IC: 1,47 a 5,45) para mortalidade em 1 ano após alta hospitalar (tendência linear $\operatorname{com} P=0,003$ ). A associação do estado nutricional com a dependência em pelo menos uma das atividades diárias foi significativa na avaliação nos primeiros 3 meses pós-alta $(\mathrm{RO}=2,81$, IC: 1,06 a 7,46) mesmo após a análise ajustada pelas variáveis citadas acima, porém não foi mantida esta associação na análise de 1 ano após alta. Também os pacientes considerados desnutridos graves pela ANS tinham maior probabilidade de encontrarse em clínicas de repouso 1 ano após a alta hospitalar $(\mathrm{RO}=3,22$, IC: 1,05 a 9,87 após análise ajustada).
Em resumo, apesar do método ter sido desenvolvido para uso em pacientes cirúrgicos, a ANS teve sua validade preditiva para morbimortalidade comprovada em diversas situações clínicas. No entanto, deve ser observada a classificação original do método, uma vez que a criação de novas categorias pode alterar sua performance.

\section{CONCLUSÃO}

Diante da falta de um método que possa ser considerado padrão ouro na avaliação nutricional, a utilização da ANS tem se mostrado boa opção, principalmente em pacientes cirúrgicos onde se deseja avaliar realmente a presença de risco nutricional pré-operatório. Por sua facilidade de execução, além da boa repetibilidade após treinamento adequado do observador, a ANS vem se tornando o método de escolha também em outras situações clínicas, seja na sua forma original, ou após adaptações. Apesar de possibilitar a participação de todos os membros da equipe multidisciplinar de terapia nutricional na realização da avaliação nutricional, é indispensável o treinamento adequado de todos os observadores que desejam praticá-la, pois a precisão do método depende da capacidade do observador em detectar as alterações nutricionais significativas através da avaliação subjetiva.

Algumas críticas a respeito dos estudos utilizados na avaliação do método, porém, devem ser feitas. A maioria deles usou a correlação com métodos objetivos para mostrar sua validade. No entanto, seria mais adequado considerar o método como variável categórica e realizar análises de variância com os demais métodos objetivos, como medidas antropométricas e testes laboratoriais.

A complementação da ANS com outras técnicas de avaliação nutricional é sugerida, uma vez que o método não possui sensibilidade adequada para identificar pequenas variações no estado nutricional e, portanto, não é indicado como método de acompanhamento da evolução do paciente em terapia nutricional.

Barbosa-Silva MCG, Barros AJD. Subjective global assessment. Part 1 - A review of its validity after two decades of use. Arq Gastroenterol 2002;39(3):181-187.

ABSTRACT - Background - The subjective global assessment is a clinical method for nutritional assessment that considers not only body composition alterations but also changes in physiological function. The method is simple, inexpensive and non-invasive, and it can be performed at bedside, and its use was described for the first time almost two decades ago. Aim - To review the validity studies of subjective global assessment described in literature in the last two decades of its use. Methods - It was performed a systematic review in MEDLINE, using "subjective global assessment" as search term and the most relevant papers were selected. Results - Being a subjective method, its precision depends on the observer's experience. Nevertheless, the method showed a good diagnostic precision when performed by trained observers. Subjective global assessment was validated by convergent validity, when this method was compared to other objective nutritional assessment methods, and by predictive validity, showing that subjective global assessment could identify patients who were at high risk for developing postoperative complications. Conclusions - Subjective global assessment has been a good option in nutritional assessment in surgical patients and some modifications have been suggested to adjust the method to other clinical situations. The observer experience is of extreme importance, since the precision of the method depends on it.

HEADINGS - Nutrition assessment. Nutrition disorders. 


\section{REFERÊNCIAS BIBLIOGRÁFICAS}

1. Abdullah MS, Wild G, Jacob V, Milford-Ward A, Ryad R, Zanaty M, Ali MH, Nahas AME. Cytocines and the malnutrition of chronic renal failure. Miner Electrolyte Metab 1997;23:237-42.

2. Allison SP. Malnutrition, disease and outcome. Nutrition 2000;16:590-3.

3. Baker JP, Detsky AS, Wesson DE, Wolman SL, Stewart S, Whitewell J, Langer B, Jeejeebhoy KN. Nutritional assessment: a comparison of clinical judgment and objective measurements. N Engl J Med 1982;306:967-72.

4. Bowers JM, Dols CL. Subjective global assessment in HIV-infected patients. J Assoc Nurses AIDS Care 1996;7:83-9.

5. Braunschweig CA. Creating a clinical nutrition registry: prospects, problems, and preliminary results. J Am Diet Assoc 1999;99:467-70.

6. Braunschweig CA, Gomez S, Sheean PM. Impact of declines in nutricional status on outcomes in adult patients hospitalized for more than 7 days. J Am Diet Assoc 2000; $100: 1316-22$

7. Campos ACL, Meguid MM. A critical appraisal of the usefulness of perioperative nutritional support. Am J Clin Nutr 1992;55:117-30.

8. Canada-USA (CANUSA) Peritoneal Dialysis Study Group. Adequacy of dialysis and nutrition in continous peritoneal dialysis: association with clinica outcomes. J Am Soc Nephrol 1996;7:198-207.

9. Coppini LZ, Waitzberg DL, Ferrini MT, Silva MLT, Gama-Rodrigues JJ, Ciosak SI. Comparação da avaliação nutricional subjetiva global x avaliação nutricional objetiva. Rev Assoc Med Bras 1995;41:6-10.

10. Corish CA. Pre-operative nutritional assessment. Proc Nutr Soc 1999;58:821-9.

11. Corish CA, Kennedy NP. Protein-energy undernutrition in hospital in-patients. Br J Nutr 2000;83:575-91.

12. Correia MITD. Avaliação nutricional subjetiva. Rev Bras Clin 1998;13:68-73

13. Correia MITD. Assessing the nutritional assessment. NCP 1999;14:142-3.

14. Covinsky KE, Martin GE, Beyth RJ, Justice AC, Sehgal AR, Landefeld CS. The relationship between clinical assessments of nutritional status and adverse outcomes in older hospitalized medical patients. J Am Geriatr Soc 1999;47:532-8.

15. Detsky AS, Baker JP, Mendelson RA, Wolman SL, Wesson DE, Jeejeebhoy KN Evaluating the accuracy of nutritional assessment techniques applied to hospitalized patients: methodology and comparisons. JPEN J Parenter Enteral Nutr 1984;8:153-9.

16. Detsky AS, Mendelson RA, Baker JP, Jeejeebhoy KN. The choice to treat all, some, or no patients undergoing gastrointestinal surgery with nutritional support: a decision analysis approach. JPEN J Parenter Enteral Nutr 1984;8:245-53.

17. Detsky AS, Baker JP, O'Rourke K, Johnston N, Whitwell J, Mendelson RA, Jeejeebhoy KN. Predicting nutrition-associated complications for patients undergoing gastrointestinal surgery. JPEN J Parenter Enteral Nutr 1987; 11:440-6.

18. Detsky AS, McLaughlin JR, Baker JP, Johnston N, Whittaker S, Mendelson RA Jeejeebhoy KN. What is subjective global assessment of nutritional status? JPEN J Parenter Enteral Nutr 1987;11:8-13.

19. Detsky AS. Nutritional status assessment: does it improve diagnostic or prognostic information? Nutrition 1991;7:37-8.

20. Detsky AS, Smalley PS, Chang J. Is this patient malnourished? JAMA 1994;271:54-8.

21. Egger NG, Carlson GL, Shaffer JL. Nutritional status and assessment of patients on home parenteral nutrition: anthropometry, bioelectrical impedance or clinical judgment? Nutrition 1999;15:1-6.

22. Ek A-C, Unosson M, Larsson J, Ganowiak W, Bjurulf P. Interrater variability and validity in subjective global assessment of elderly patients. Scan J Caring Sci $1996 ; 10: 163-8$

23. Enia G, Sicuso C, G GA, Zoccali C, Pustorino D, Biondo A. Subjective global assessment of nutrition in dialysis patients. Nephrol Dial Transplant 1993;8:1094-8.
24. Fiaccadori E, Lombardi M, Leonardi S, Rotelli CF, Tortorella G, Borghetti A. Prevalence and clinical outcome associated with preexisting malnutrition in acute renal failure: a prospective cohort study. J Am Soc Nephrol 1999;10:58193.

25. Foulks CJ. What is Subjective Global Assessment? NCP 2001;16:263

26. Green CJ. Existence, causes and consequences of disease-related malnutrition in the hospital and the community, and clinical and financial benefits of nutritional intervention. Clin Nutr 1999;18:3-28.

27. Hasse J, Strong S, Gorman MA, Liepa G. Subjective global assessment: alternative nutrition-assessment technique for liver-transplant candidates. Nutrition 1993;9:339-43.

28. Hill GL. Body composition research: implications for the practice of clinical nutrition. JPEN J Parenter Enteral Nutr 1992;16:197-218.

29. Hirsch S, Obaldia Nd, Petermann M, Rojo P, Barrientos C, Iturriaga H, Bunou D. Subjective global assessment of nutritional status: further validation. Nutrition 1991;7:35-8.

30. Irving GF, Olsson BA, Cederholm T. Nutritional and cognitive status in elderly subjects living in service flats, and the effect of nutrition education on personnel. Gerontology 1999;45:187-94

31. Jeejeebhoy KN, Detsky AS, Baker JP. Assessment of nutritional status. JPEN J Parenter Enteral Nutr 1990;14:193S-6S.

32. Jeejeebhoy KN. Nutritional assessment. Nutrition 2000;16:585-90

33. Jones CH, Newstead CG, Will EJ, Smye SW, Davison AM. Assesment of nutritional status in CAPD patients: serum albumin is not a useful measure. Nephrol Dial Transplant 1997;12:1406-13

34. Kalantar-Zadeh K, Kleiner M, Dunne E, Ahern K, Nelson M, Koslowe R, Luft FC. Total iron-binding capacity-estimated transferrin correlates with the nutritional subjective global assessment in hemodialysis patients. Am J Kidney Dis 1998;31:263-72.

35. Kalantar-Zadeh K, Kleiner M, Dunne E, Lee GH, Luft FC. A modified quantitative subjective global assessment of nutrition for dialysis patients. Nephrol Dia Transplant 1999;14:1732-8.

36. Klein S, Kinney J, Jeejeebhoy KN, Alpers D, Hellerstein M, Murray M, Twomey P. Nutrition support in clinical practice: a review of published data and recommendations for future research directions. JPEN J Parenter Enteral Nutr 1997;21:133-56.

37. McClave SA, Snider HL, Spain DA. Preoperative issues in clinical nutrition. Chest 1999;115:64S-70S.

38. Naber THJ, Schermer T, Bree Ad, Nusteling K, Eggink L, Kruimel JW, Bakkeren J, Heereweld Hv, Katan MB. Prevalence of malnutrition in nonsurgical hospitalized patients and its association with disease complications. Am J Clin Nutr 1997;66:1232-9.

39. Niyongabo T, Melchior JC, Henzel D, Bouchaud O, Larouze B. Comparison of methods for assessing nutritional status in HIV-infected adults. Nutrition 1999; 15:740-3.

40. Ottery FD. Nutritional oncology: a proactive, integrated approach to the cance patient. In: Shikora SA, Blackburn GL, editors. Nutrition support: theory and therapeutics. New York: Chapman \& Hall; 1997. p.395-409.

41. Pikul J, Sharpe MD, Lowndes R, Ghent CN. Degree of preoperative malnutrition is predictive of postoperative morbidity and mortality in liver transplant recipients. Transplantation 1994;57:469-72.

42. Schneider SM, Hebuterne X. Use of nutritional scores to predict clinical outcomes in chronic diseases. Nutr Rev 2000;58:31-8.

43. The Veterans Affairs Total Parenteral Nutrition Cooperative Group. Perioperative total parenteral nutrition in surgical patients. N Engl J Med 1991;325:525-32.

44. Waitzberg DL, Caiaffa WT, Correia MITD. Hospital malnutrition: the Brazilian national survey (IBRANUTRI): a study of 4000 patients. Nutrition $2001 ; 17: 573-80$. 